Article

\title{
Spatial and Temporal Optimization Strategy for Plug-In Electric Vehicle Charging to Mitigate Impacts on Distribution Network
}

\author{
Lili Gong ${ }^{1}$, Wu Cao ${ }^{1}$, Kangli Liu ${ }^{1}{ }^{\mathbb{D}}$, Jianfeng Zhao ${ }^{1, *}$ and Xiang $\mathrm{Li}^{2}$ \\ 1 School of Electrical Engineering, Southeast University, Nanjing 210096, China; lili_gong@seu.edu.cn (L.G.); \\ caowu_ee@seu.edu.cn (W.C.); kangcumt@yeah.net (K.L.) \\ 2 LiuZhou Power Supply Bureau, GuangXi Power Grid Co., Ltd., Liuzhou 545006, China; \\ li_xiang.lzg@gx.csg.cn \\ * Correspondence: jianfeng_zhao@seu.edu.cn; Tel.: +86-13951943657
}

Received: 26 April 2018; Accepted: 21 May 2018; Published: 29 May 2018

\begin{abstract}
The large deployment of plug-in electric vehicles (PEVs) challenges the operation of the distribution network. Uncoordinated charging of PEVs will cause a heavy load burden at rush hour and lead to increased power loss and voltage fluctuation. To overcome these problems, a novel coordinated charging strategy which considers the moving characteristics of PEVs is proposed in this paper. Firstly, the concept of trip chain is introduced to analyze the spatial and temporal distribution of PEVs. Then, a stochastic optimization model for PEV charging is established to minimize the distribution network power loss (DNPL) and maximal voltage deviation (MVD). After that, the particle swarm optimization (PSO) algorithm with an embedded power flow program is adopted to solve the model, due to its simplicity and practicality. Last, the feasibility and efficiency of the proposed strategy is tested on the IEEE 33 distribution system. Simulation results show that the proposed charging strategy not only reduces power loss and the peak valley difference, but also improves voltage profile greatly.
\end{abstract}

Keywords: plug-in electric vehicles; coordinated charging; distribution network; trip chain; particle swarm optimization; national household trip survey data

\section{Introduction}

The intensification of environmental pollution has drawn people's attention to renewable energy generation, electric vehicles (EVs) and some other energy scavenging technologies [1]. As a clean, efficient and eco-friendly means of transportation, plug-in electric vehicles (PEVs) are widely used throughout the whole world. However, the high penetration of PEVs will lead to large amount of power consumption, which will increase the load burden of the distribution network [2]. Meanwhile, the uncoordinated charging of PEVs will also bring negative impacts on the distribution network, such as overloading, increased power loss, voltage drop and so on [3]. In [4], the specific impacts including power loss and voltage deviation have been discussed under uncoordinated and coordinated charging scenarios, the results showed that the uncoordinated charging of plug-in hybrid electric vehicles (PHEVs) reduces the efficiency of the distribution network. The potential impacts from various levels were investigated in [5], where simulation results illustrated that the uncontrolled charging of EVs could also result in levels of unbalance. The power quality impact assessment was conducted in [6], which considered the random operating characteristics of PHEVs. A stochastic approach based on actual measurements and survey data was proposed in [7] to analyze the voltage and congestion impacts of electric vehicles (EVs), the conclusion was that the security problems caused by EVs can be 
mitigated by smart charging. Therefore, the proper control of PEVs is critical to ensure the safe and economical operation of the distribution network.

Much research has focused on the coordinated charging of PEVs. In [8], a centralized control charging method has been proposed to maximize the total power that could be transmitted to EVs by controlling the charging rates. In [9], a decentralized charging strategy was proposed to fill load valleys, whereby the charging profile of each EV was updated according to the delivered control signals. In [10], an intelligent pricing scheme was proposed to coordinate PEV charging, in which the price and quantity information were sent to the load aggregators by the system operator. In [11], a new application system was designed for EV management in a smart distribution network. Environmental issues, economic issues and various driving patterns of EVs owners were all considered in the proposed multi-objective optimization model. The benders decomposition technique was adopted to solve the problem, and results showed that this method has good convergence. In [12], EV charging was formulated as a linear optimization problem taking distribution network constraints into account, which was suitable for charging large numbers of EVs. In [13], two charging pricing mechanisms under non-cooperative and cooperative scenarios were presented to guide the charging schedules of EV owners. The coordinated charging strategies for different scenarios were designed according to different requirements, which reduced the computational complexity and guaranteed quick convergence. In [14], a novel valley-filling strategy for large-scale EVs was proposed to mitigate negative impacts on the power grid. It defined two important indexes to select proper target time slots and determine the charging priority of EVs. The results showed that the centralized charging strategy was highly efficient. In [15], coordination of PEVs was proposed to achieve valley filling and charging cost reduction, coupling the customer and grid-based objectives prominently.

Although these charging strategies are effective for solving the corresponding problems, the changing characteristics that lead to the random charging behavior of PEVs are more or less ignored. Compared with previous research, this paper proposes a novel charging strategy which considers the moving characteristics of PEVs to mitigate impacts on distribution network. First, the spatial-temporal distribution of PEVs is discussed based on trip chain and national household trip survey (NHTS) data. Second, for both economic and power quality concern, a stochastic optimization model for PEV charging is established to minimize the distribution network power loss (DNPL) and maximal voltage deviation (MVD). Then, the particle swarm optimization (PSO) algorithm with an embedded power flow program is adopted to solve the model by shifting the charging load of PEVs to different locations. Finally, simulations are conducted to verify the proposed model and strategy.

The rest of this paper is organized as follows: Section 2 describes the spatial and temporal distribution of PEVs. Section 3 presents the optimization model and solving method of the proposed charging strategy. In Section 4, the simulation results and discussions are presented. Finally, Section 5 draws the conclusions.

\section{The Spatial and Temporal Distribution of PEVs}

\subsection{PEVs' Daily Movement}

Due to the mobility of PEVs and users' travel habits, the charging behavior of PEVs is full of randomness. To obtain the spatial and temporal distribution of PEVs, the concept of the trip chain is introduced here to analyze users' travel behavior [16]. According to national household trip survey (NHTS) data [17], the trip destinations of PEVs can be classified into four types: Home (H), Work (W), Public leisure $(\mathrm{P})$ and Other transactions $(\mathrm{O})$. The typical daily movement of PEVs based on trip chain is shown in Figure 1. 

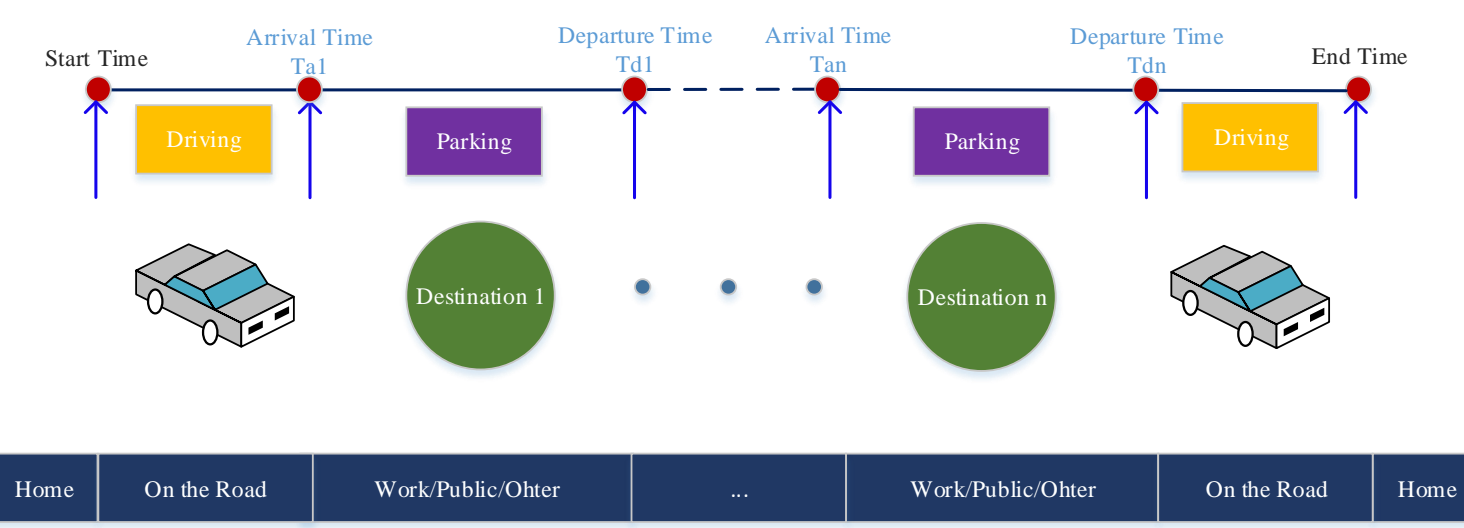

Figure 1. The typical daily movement of PEVs based on trip chain.

\subsection{Construction of Trip Chain and the Transition Probability}

Studies reveal that drivers usually both start and end all their trips at home. In addition, for private cars, the average length of trip chain is 3.02 [18]. Therefore, no more than three trip destinations are considered in this paper and the starting and terminal point is specified as home. Therefore, the trip chains of PEVs are constructed only between these four zones, as shown in Table 1.

Table 1. Trip chains of PEVs.

\begin{tabular}{cccc}
\hline Type & \multicolumn{3}{c}{ Trip Chain } \\
\hline 2 trips & H-W-H & H-P-H & H-O-H \\
\hline \multirow{3}{*}{3 trips } & H-W-P-H & H-P-W-H & H-P-P-H \\
& H-W-O-H & H-O-W-H & H-O-P-H \\
& H-W-W-H & H-P-O-H & H-O-O-H \\
\hline
\end{tabular}

In Table 1, a trip represents the movement between the original and final locations. For example, a trip from home to public place is defined as H-P. It is assumed that the movement of PEVs between different zones conforms to the discrete markov process [19], then transitional probability from state $D_{i}$ to state $D_{j}$ is given by (1):

$$
p\left(E_{D_{i}} \rightarrow E_{D_{j}}\right)=p\left(E_{D_{i}} \mid E_{D_{i}}\right)=p_{D_{i} D_{j}}
$$

Correspondingly, the transition probability matrix of PEVs between zones in this paper can be obtained as:

$$
\begin{array}{ccccc}
\mathrm{H} & \mathrm{P} & \mathrm{W} & \mathrm{O} \\
\mathrm{H} \\
\mathrm{P} \\
\mathrm{W} \\
\mathrm{O}
\end{array} \quad\left(\begin{array}{llll}
p_{H H} & p_{H P} & p_{H W} & p_{H O} \\
p_{P H} & p_{P P} & p_{P W} & p_{P O} \\
p_{W H} & p_{W P} & p_{W W} & p_{W O} \\
p_{O H} & p_{O P} & p_{O W} & p_{O O}
\end{array}\right)
$$

where the transition probability of each trip can be obtained by NHTS data.

\subsection{Charging Load of PEVS}

The charging load of PEVs depends on many important factors, such as charging location, charging mode and the number of PEVs. As mentioned in Sections 2.1 and 2.2, the trip destination appearing in a trip chain indicates that users may stay and charge at the corresponding charging location. However, it is unreasonable to assume that PEVs could be charged anywhere, because of limited charging facilities. Therefore, the charging location is specified as the charging station in 
different zones, including residential charging station (RCS), working area charging station (WCS), public leisure area charging station (PCS) and other regional charging station (OCS).

Generally, the PEV charging mode can be divided into slow charging mode and fast charging mode [20]. The slow charging mode is usually adopted at home or the workplace, with low charging power and long charging duration. It is assumed that slow charging mode is used at RCS and WCS. The fast charging mode provides a high charging power, which is also called urgent charging. In fast charging mode, PEVs can be fully charged in a short time. Therefore, the fast charging mode is adopted in PCS and OCS.

After the determination of charging location and charging mode, the charging load can be obtained by (3):

$$
P_{Z, t}=\sum_{n e=1}^{N_{Z}} P_{C h} s_{n e, t}
$$

where $P_{Z, t}$ is the load of charging station $Z$ at time $t, Z \in\{$ RCS, WCS, PCS, OCS $\} ; N_{Z}$ is the number of PEVs in charging station $Z ; P_{C h} \in\left\{P_{C h F}, P_{C h S}\right\}$ is the charging power, $P_{C h F}$ is the fast charging power, $P_{C h S}$ is the slow charging power; $S_{n e, t}$ is the charging state of the ne th PEV at time $t$, if charging, $S_{n e, t}=1$,else, $S_{n e, \mathrm{t}}=0$.

In this paper, the number of PEVs in each charging station is not deterministic, it varies in a range, and depends on the demand of users. Its formula is as follows:

$$
N_{Z}=N_{E V} p_{Z}, p_{Z, \min } \leq p_{Z} \leq p_{Z, \max }
$$

where $N_{E V}$ is the total number of PEVs in the distribution network; $p_{Z}$ is the parking probability for charging station $Z ; p_{Z \text {, max }}$ and $p_{Z \text {,min }}$ are the maximum and minimum value of $p_{Z}$, which can be obtained through the analysis of historical information and the transition probability.

The charging condition of PEVs is set as follows:

$$
\begin{cases}T_{a}<t<T_{a}+T_{c}, & s_{n e, t}=1 \\ \text { else, } & s_{n e, t}=0\end{cases}
$$

where $T_{a}$ is the arrival time, and the arrival time for each charging station is different, but each follows the normal distribution [21] denoted by $T_{a} \sim N\left(\mu_{T}, \sigma_{T}^{2}\right), \mu_{T}$ is the average value and $\sigma_{T}$ is the standard deviation. In this paper, it is assumed that PEVs can be charged immediately when arriving at the station. Therefore, the initial charging time of PEV is the arrival time. $T_{c}$ is the charging duration, which can be calculated by (6):

$$
T_{c}=\frac{\left(S O C_{f}-S O C_{s}\right) B_{c}}{\lambda P_{C h}}
$$

where $S O C_{s}$ and $S O C_{f}$ are the initial and final state of charge (SOC), respectively; $B_{c}$ is the battery capacity, $\lambda$ is the charging efficiency.

On this basis, the spatial-temporal load model of PEVs that can be directly applied in the coordinated charging procedure is established, and the flow chart is shown in Figure 2. 


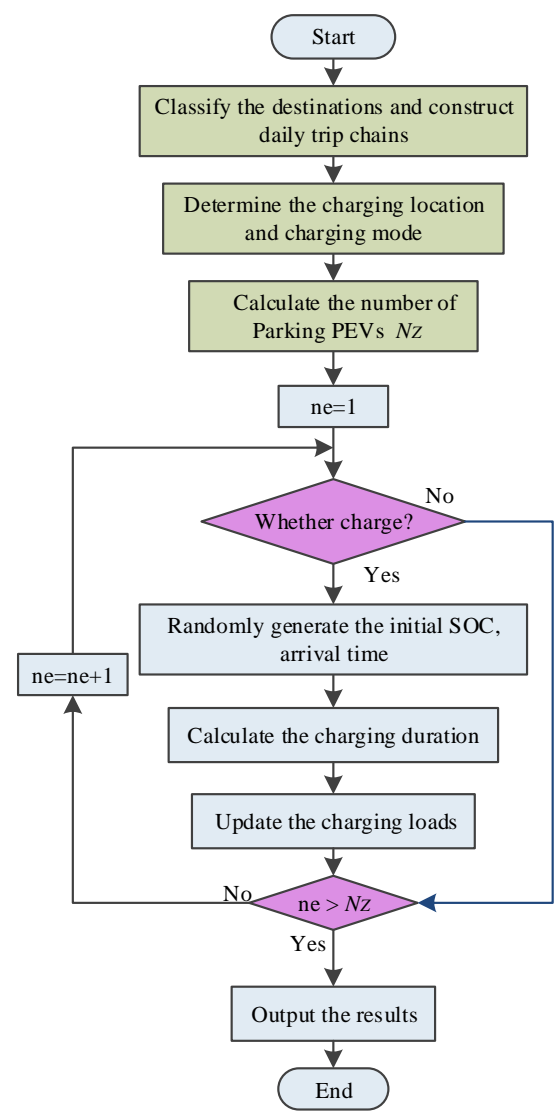

Figure 2. The determination of the spatial and temporal distribution of PEVs.

\section{Problem Formulation and Methodology}

In this paper, the specific spatial and temporal optimal charging strategy of PEVs is proposed to mitigate impacts on distribution network, including power loss reduction and voltage profile improvement. It considers the moving characteristics of PEVs and expands the charging location from a single residential charge to a full-area charge. Based on the analysis of the spatial and temporal distribution of PEVs, the optimal strategy is achieved by adjusting the number of PEVs to shift charging load to different areas.

\subsection{Objective Function}

Power loss is an important economic concern of distribution networks, and occupies more than $40 \%$ of the total network loss [22]. It is necessary to minimize power loss for the economic operation of the distribution network. The expression of DNPL is as follows:

$$
D N P L=\sum_{t=1}^{T t} \sum_{l=1}^{N b r a n c h} R_{l} I_{l, t}^{2}
$$

where $T_{t}$ is the total number of charging periods, and is set as $24 ; N_{b r a n c h}$ is the total number of branches; $R_{l}$ is the resistance of branch $l ; I_{l, t}$ is the current of branch $l$ at time $t$.

From the distribution network operator, the voltage deviation partly represents the power quality concern, which is essential to the safe operation of the distribution network [23]. It is important to avoid large voltage deviations. The expression of MVD is as follows:

$$
M V D=\max _{i=1,2, \ldots, \text { Nnode }}\left(\max _{t=1,2, \ldots, T t}\left(\left|\frac{U_{i, t}-U_{N}}{U_{N}}\right|\right)\right)
$$


where $N_{\text {node }}$ is the total number of nodes; $U_{i, t}$ is the voltage of node $i$ at time $t ; U_{N}$ is the rated voltage.

The problem involves multi-objective optimization, and two optimization targets are considered in the proposed model. Although some algorithms, such as the Pareto solution set method [24] and the lexicographic method [25], are efficient for solving multi-objective optimization, the linear weighting-sum method [26] is used here for its better practicability and convenience. The two optimization goals are first normalized to the same range and then processed into a single objective function. The total objective function is formulated as follows:

$$
\min F=\min \left(\alpha f_{1}+\beta f_{2}\right)
$$

where $f_{1}$ is the normalized maximal voltage deviation; $f_{2}$ is the normalized power loss; $\alpha$ and $\beta$ are the weight coefficients.

\subsection{Determination of Weight Coefficient}

The judgment matrix method is a reliable quantification method to determine the weight coefficients [27]. It classifies all objectives into different scales according to importance, and then form a square matrix based on standardized scores. Usually, the scales 1, 2, 3, .., 9 and their reciprocals are used to represent the relative importance. The meanings are shown in Table 2 [28].

Table 2. The rule of constructing the judgment matrix.

\begin{tabular}{cc}
\hline Scale & Meaning \\
\hline 1 & The two goals are of equal importance \\
3 & One is slightly more important than the other \\
5 & One is obviously more important than the other \\
7 & One is strongly more important than the other \\
9 & One is extremely more important than the other \\
\hline
\end{tabular}

In this paper, the DNPL is considered slightly more important than the MVD. Therefore, the judgment matrix is formed as:

$$
J=\left[\begin{array}{cc}
1 & 1 / 3 \\
3 & 1
\end{array}\right]
$$

By solving the eigenvalues and characteristic vectors of the matrix [29], the weight coefficients of each subgoal can be obtained $\alpha=0.75, \beta=0.25$.

\subsection{System Constraints}

The first equality constraint is set for the power flow, which is as follows:

$$
\left\{\begin{array}{c}
P_{i, t}=U_{i, t} \sum_{j=1}^{\text {Node }} U_{j, t}\left(G_{i j} \cos \delta_{i j, t}+B_{i j} \sin \delta_{i j, t}\right) \\
Q_{i, t}=U_{i, t} \sum_{j=1}^{\text {Node }} U_{j, t}\left(G_{i j} \sin \delta_{i j, t}-B_{i j} \cos \delta_{i j, t}\right)
\end{array}\right.
$$

where $P_{i, t}$ is the active power of node $i$ at time $t ; Q_{i, t}$ is the reactive power of node $i$ at time $t ; U_{i, t}$ and $U_{j, t}$ are the voltage amplitude of node $i$ and node $j$ at time $t$, respectively; $\delta_{i j, t}$ is the voltage angle between node $i$ and node $j$ at time $t ; G_{i j}$ is the conductance between node $i$ and node $j ; B_{i j}$ is the susceptance between node $i$ and node $j$.

The second constraint is set for the node voltage, which is as follows:

$$
U_{\min } \leq U_{i, t} \leq U_{\max }
$$


where $U_{\min }$ is the minimum value of node voltage, which is set as $0.9 \mathrm{pu} ; U_{\max }$ is the maximum value of node voltage, which is set as $1.1 \mathrm{pu}$.

The third constraint is set for PEVs:

$$
\left\{\begin{array}{l}
p_{z, \min } N_{E V} \leq N_{Z} \leq p_{z, \max } N_{E V} \\
N_{P C S}+N_{W C S}+N_{O C S} \leq N_{R C S}
\end{array}\right.
$$

\subsection{Solving Method}

The coordinated charging of PEVs is a complex nonlinear optimization problem, which can be solved by many kinds of modern heuristics algorithm, such as ant colony algorithms [30], genetic algorithms [31] and PSO algorithms [32]. Due to its simplicity and convenience, the PSO algorithm with an embedded power flow program is applied to solve the optimization problem. The inner-layer power flow calculation is carried out by back/forward sweep method, which is widely used in power systems. According to the calculated fitness function, the outer PSO algorithm constantly searches for the optimal results until the termination condition is satisfied. The specific steps are as follows:

Step 1: Input raw data, including parameters of the distribution network and PEVs.

Step 2: Specify some parameters associated with PSO, such as the population size $N_{S}$, the dimension of particle $D$, the maximum number of iterations $M$.

Step 3: Randomly generate the initial particle swarm. In the optimization model of this paper, once the parking probability of PEVs in area $Z$ is determined, the number of PEVs parked at different charging stations is also obtained. Therefore, the parking probability of PEVs is set as unknown quantity $X$ in the PSO algorithm. Every particle has $D$ dimensions, each dimension represents the number of PEVs parking at the corresponding charging station besides RCS. Because for all PEVs in this paper, the last trip destination is specified as home, the parking probability in RCS is a constant.

Step 4: Carry out the power flow calculation to initialize the fitness function. In the inner-layer program, set the sample array of the charging load of different charging stations including Ppcs [T], $P_{R} c s[T], P_{O} c s[T], P_{W} c s[T]$ and perform the power flow calculation, $T=24$. During the calculation, the power flow equation and voltage constraint would be satisfied automatically. In addition, the load distribution of PEVs is also initialized.

Step 5: Update the inertia weight and learning factors of particle swarm by (14):

$$
\left\{\begin{array}{l}
\omega=\omega_{\max }-\left(\omega_{\max }-\omega_{\min }\right) m / M \\
c_{a}=c_{a i n i}+\left(c_{a f i n}-c_{a i n i}\right) m / M \\
c_{b}=c_{b i n i}+\left(c_{b f i n}-c_{b i n i}\right) m / M
\end{array}\right.
$$

where $\omega$ is the inertia weight, $\omega_{\max }$ is the maximum value of $\omega, \omega_{\min }$ is the minimum value of $\omega$; $c_{a}$ and $c_{b}$ are two learning factors; $c_{a i n i}$ is initial value of $c_{a}$ and $c_{a f i n}$ is the final value of $c_{a} ; c_{b i n i}$ and $c_{b f i n}$ are the initial and final value of $c_{b}$, respectively.

Step 6: Update the velocity and position of particle swarm by (15):

$$
\left\{\begin{aligned}
v_{n d}(m+1) & =\omega v_{n d}(m)+c_{a} \operatorname{rand}_{a}\left(x_{p n}(m)-x_{n d}(m)\right) \\
& +c_{b} \operatorname{rand}_{b}\left(x_{g}(m)-x_{n d}(m)\right) \\
x_{n d}(m+1) & =x_{n d}(m)+v_{n d}(m+1)
\end{aligned}\right.
$$

where $x_{n d}(m)$ and $v_{n d}(m)$ are the position and velocity along dimension $d$ of particle $n$ in iteration $m$, respectively; $x_{p n}(m)$ is the best position of each particle; $x_{g}(m)$ is the best position among all the particles in the population; rand $_{a}$ and rand $_{b}$ are random numbers within a range of $[0,1]$. 
Step 7: To avoid the particle flying beyond its scope of velocity and position, the constraints of charging demand are handled as follows:

$$
\begin{gathered}
v_{n d}(m)=\left\{\begin{array}{c}
v_{\min }, v_{n d}(m) \leq v_{\min } \\
v_{\max }, v_{n d}(m) \geq v_{\max }
\end{array}\right. \\
x_{n d}(m)=\left\{\begin{array}{l}
x_{\min }, x_{n d}(m) \leq x_{\min } \\
x_{\max }, x_{n d}(m) \geq x_{\max }
\end{array} \text { set } v_{n d}(m)=-v_{n d}(m)\right. \\
x_{n 3}(m)=p_{R C S}-x_{n 2}(m)-x_{n 1}(m), \sum_{d=1}^{D} x_{n d}(m)>p_{R C S}
\end{gathered}
$$

where $v_{\min }$ and $v_{\max }$ are the minimum and maximum value of velocity, respectively; $x_{\max }$ is the maximum value of position, $x_{\min }$ is the minimum value of position, $p_{R C S}$ is the parking probability for RCS.

Step 8: Carry out the power flow calculation again, update the fitness function. During the power flow calculation, the charging load of PEVs in different charging stations is also updated.

Step 9: Repeat Steps 5-8 for $M$ times.

Step 10: Output the best position as the optimal solution, then we can obtain the optimal charging load of PEVs.

The whole process of coordinated charging of PEVs based on PSO algorithm is shown in Figure 3.

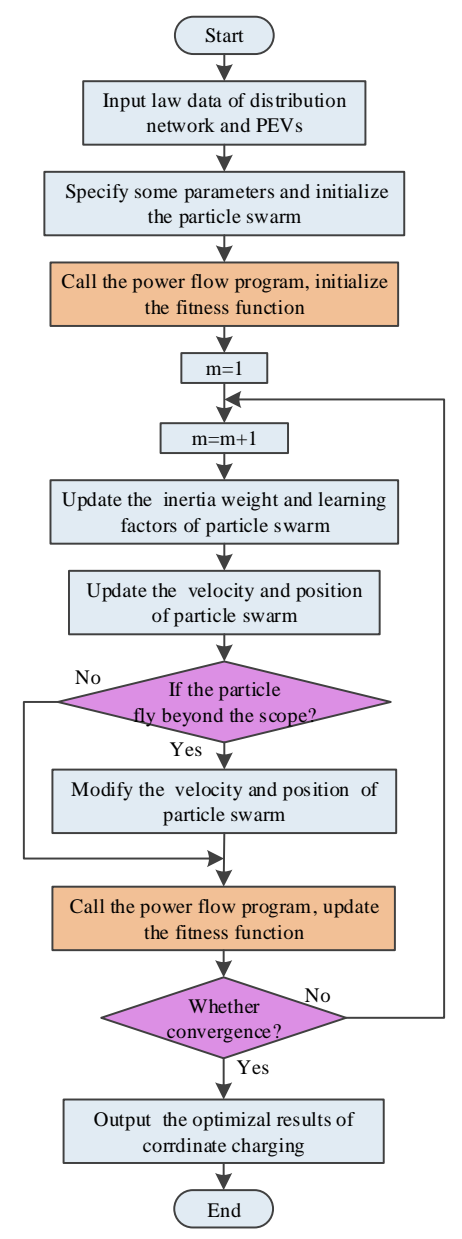

Figure 3. The coordinated charging of PEVs based on the PSO algorithm. 


\section{Case Study}

\subsection{Case Description}

In this paper, the IEEE 33 distribution system is used to demonstrate the proposed model and method. The system structure diagram is shown in Figure 4. The PCS, RCS, WCS and OCS are located at node 5, 8, 12 and 29, respectively. For each node of the IEEE 33 system, the load data is from the measured data of a real-urban area in China [33]. In addition, the typical daily load curve is shown in Figure 5. For all kinds of charging stations, $S O C_{s}$ obeys the normal distribution $\mathrm{N}(0.5,0.1), S O C_{f}$ is set as 1 , and the relevant parameters of the PEVs [34] and the PSO [35,36] algorithm are shown in Table 3. For better comparison, five different cases are described as follows:

Case 1: No PEVs connected to the distribution network.

Case 2: Random uncoordinated PEV charging may occur anytime and all PEVs charge only at RCS.

Case 3: The coordinated charging strategy is applied here and the charging probabilities at RCS, WCS, PCS and OCS are set as $0.5,0.3,0.1$ and 0.1 , respectively. Here, only DNPL is selected as the optimization objective.

Case 4: All the conditions are set the same as case 3, but only MVD is selected as the optimization objective.

Case 5: The multi-objective optimization under coordinated charging is conducted.

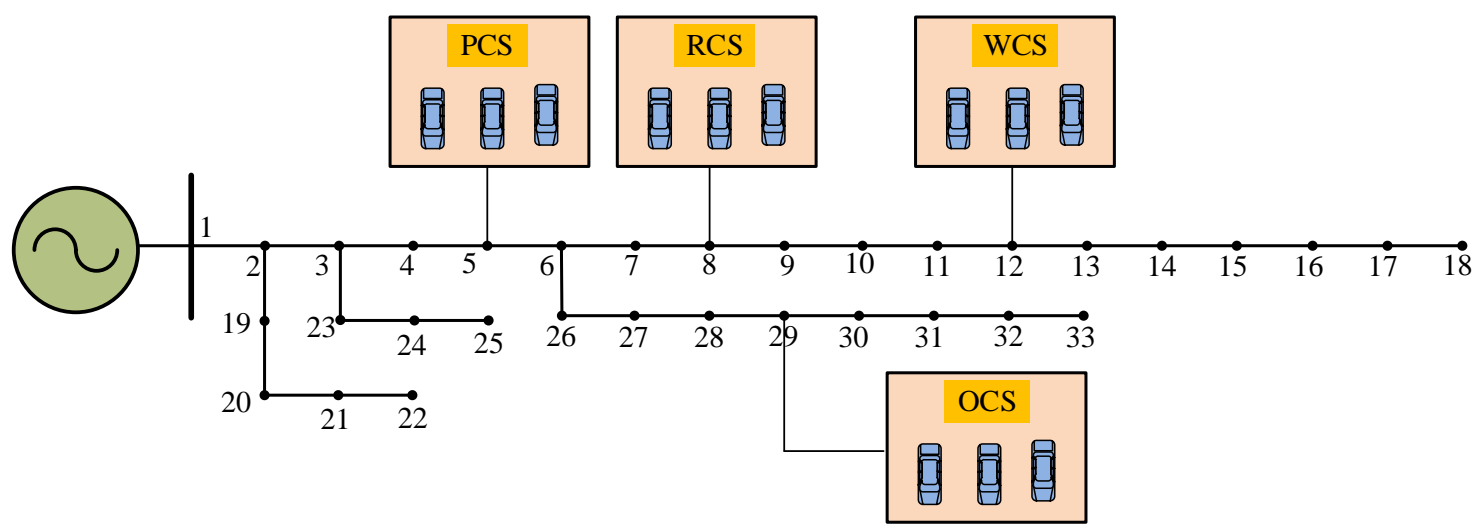

Figure 4. The structure diagram of the IEEE 33 distribution system.

Table 3. Parameters of the simulation.

\begin{tabular}{ccc}
\hline Part & Quantity & Value \\
\hline \multirow{4}{*}{ PEV } & total number of PEVs $N_{E V}$ & 500 \\
& battery capacity $B_{c} / \mathrm{kWh}$ & 30 \\
charging efficiency $\lambda$ & $90 \%$ \\
& fast charging power $P_{C h F} / \mathrm{kW}$ & 45 \\
slow charging power $P_{C h S} / \mathrm{kW}$ & 7 \\
\hline population size $N_{s}$ & 50 \\
PSO & maximum number of iterations $M$ & 200 \\
& dimension $D$ & 3 \\
& the maximum value of $\omega$ & 0.9 \\
& the minimum value of $\omega$ & 0.4 \\
& the initial value of learning factor $c_{a}$ & 2.5 \\
the final value of learning factor $c_{a}$ & 0.5 \\
the initial value of learning factor $c_{b}$ & 0.5 \\
the final value of learning factor $c_{b}$ & 2.5 \\
\hline
\end{tabular}




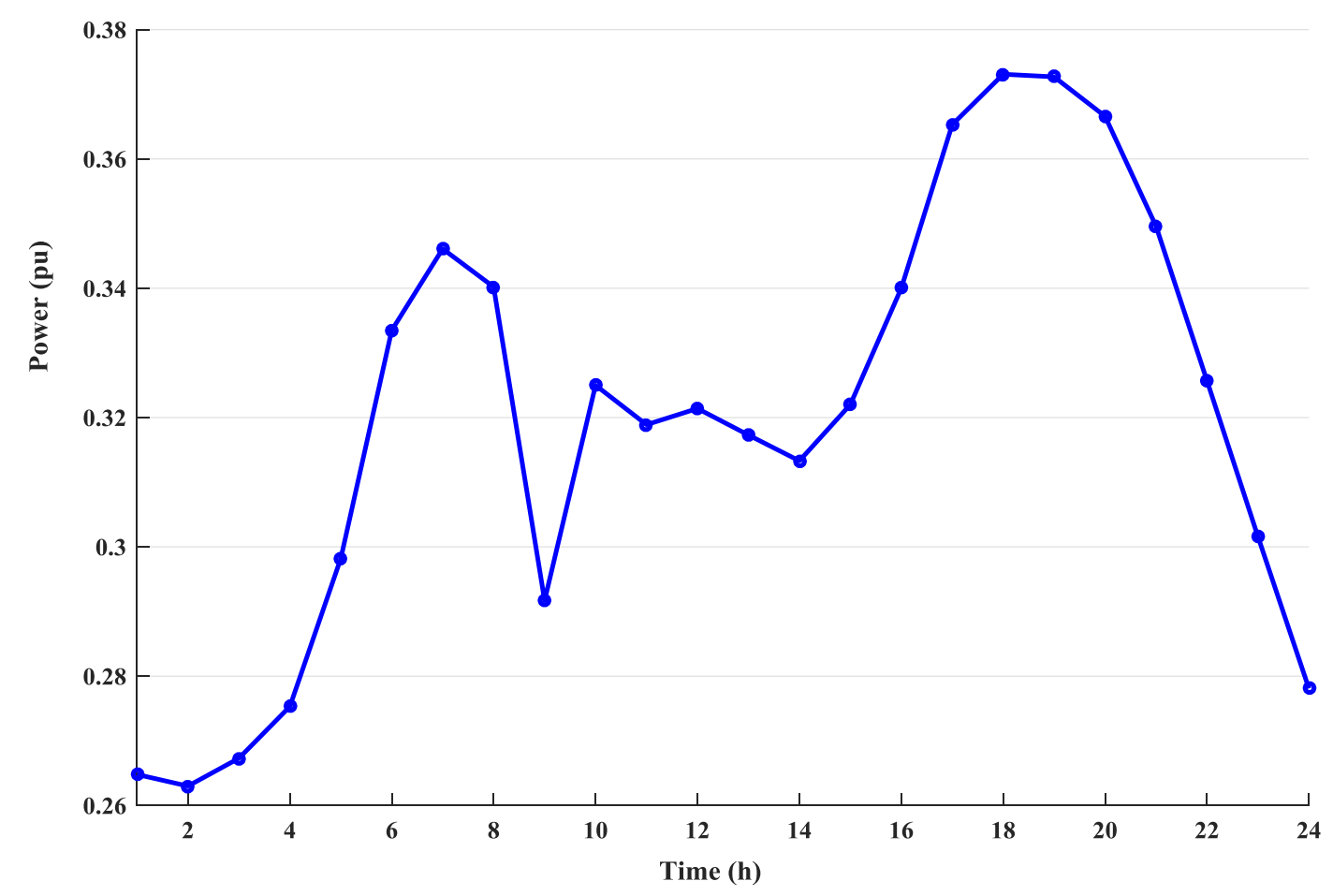

Figure 5. Typical daily load curve.

\subsection{Parameter Calculation}

The NHTS 2009 database has abundant trip records of residents. For each trip, the important information including trip destination, arrival time, departure time and driving distance are all provided. The transition probability of each trip is calculated by statistical data, which is shown in Table 4. By using the curve-fitting toolbox in MATLAB, the arrival time in different charging location is fitted with normal distribution, which is shown in Table 5 .

Table 4. Parameters of transition probability.

\begin{tabular}{cccccc}
\hline Parameters & Zone & H & P & W & O \\
\hline \multirow{4}{*}{ transition probability } & H & 0.0028 & 0.5006 & 0.261 & 0.2356 \\
& P & 0.4032 & 0.513 & 0.0206 & 0.0632 \\
& W & 0.5797 & 0.3087 & 0.0397 & 0.0719 \\
& O & 0.4582 & 0.3617 & 0.0279 & 0.1523 \\
\hline
\end{tabular}

Table 5. Parameters of arrival time.

\begin{tabular}{cccc}
\hline Parameters & Zone & Mean & Standard Deviation \\
\hline & $\mathrm{H}$ & 17.47 & 3.41 \\
arrival time $/ \mathrm{h}$ & $\mathrm{P}$ & 8.8214 & 2.7897 \\
& $\mathrm{~W}$ & 13.7059 & 3.2416 \\
& $\mathrm{O}$ & 12.605 & 3.8663 \\
\hline
\end{tabular}

\subsection{Results Analysis}

\subsubsection{Convergence Analysis}

The optimization is repeated 50 times, and the results are shown in Table 6. Over 50 runs, the final simulation results are chosen based on the best fitness value, which is closest to the average value in 
Table 6. Figure 6 shows the convergent curve of the PSO algorithm. The calculation time in all cases is less than $120 \mathrm{~s}$. Therefore, the PSO algorithm finds the best fitness values with fast convergence rate, which shows the superiority of the algorithm.

Table 6. The objective function value of PSO.

\begin{tabular}{ccc}
\hline Parameters & Mean & Variance \\
\hline Case 3 & 0.3358 & $5.3096 \times 10^{-7}$ \\
Case 4 & 0.3136 & $3.6667 \times 10^{-7}$ \\
Case 5 & 0.3319 & $6.7078 \times 10^{-7}$ \\
\hline
\end{tabular}
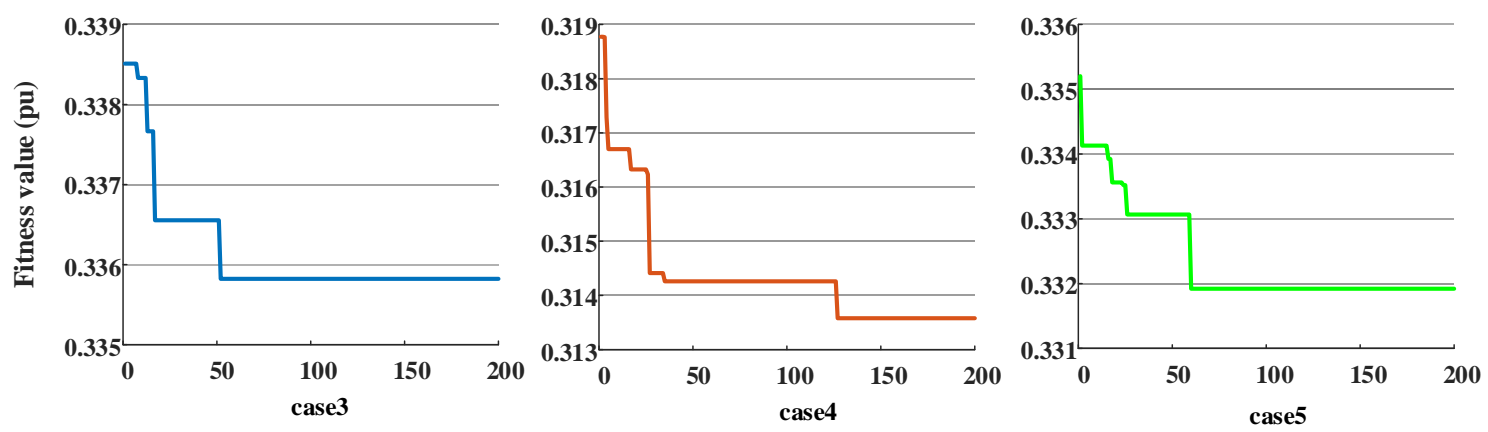

Iterations

Figure 6. Fitness function convergent curve.

The values for the obtained parking probabilities in PSO are shown in Table 7. In addition, according to Table 1 and the transition probability shown in Table 4, the parking probability for RCS can be obtained as $p_{R C S}=0.6866$, which is the same value in different cases.

Table 7. The parking probability obtained in PSO.

\begin{tabular}{cccc}
\hline Parameters & $p_{\text {PCS }}$ & $p_{\text {WCS }}$ & $p_{\text {OCS }}$ \\
\hline Case 3 & 0.2432 & 0.1522 & 0.1429 \\
Case 4 & 0.2474 & 0.197 & 0.1708 \\
Case 5 & 0.2369 & 0.1796 & 0.1481 \\
\hline
\end{tabular}

\subsubsection{Objective Functions Analysis}

The simulation results of DNPL and MVD in different cases are shown in Table 8. From Table 8, it is observed that compared with case 1, the DNPL and MVD in case 2 are both greatly increased with uncoordinated PEVs connected to the distribution network. To mitigate these negative impacts, the coordinated charging strategy is adopted in case 3-case 5. In case 3, the DNPL is maximally reduced to $3.358 \mathrm{MW}$, and in case 4, the MVD is maximally reduced to $0.3136 \mathrm{pu}$. In case 5, both the DNPL and MVD are taken as the objective functions with a weight ratio of 3:1. Compared with case 2 , the DNPL is reduced to $3.372 \mathrm{MW}$ and the MVD is reduced by $10.5 \%$, which achieves satisfactory result of both DNPL and MVD.

Table 8. Objective function values in different cases.

\begin{tabular}{cccccc}
\hline Parameters & Case $\mathbf{1}$ & Case 2 & Case 3 & Case 4 & Case 5 \\
\hline DNPL $(\mathrm{MW})$ & 3.198 & 3.45 & 3.358 & 3.403 & 3.372 \\
MVD $(\mathrm{pu})$ & 0.2935 & 0.3531 & 0.3196 & 0.3136 & 0.3161 \\
\hline
\end{tabular}


Figures 7 and 8 show the daily power loss and maximal voltage deviation, it can be found that during the peak period of load demand, both the power loss and maximal voltage deviation reach the maximum value, which increases the operation risk. From Figure 9, it can be observed that the power loss of every branch in the distribution network is very small after optimization, which avoids obvious economic loss. Figure 10 shows the node voltage of the distribution network in case 5 . In Figure 10, the voltage of each node at each moment is between $0.95 \mathrm{pu}$ and $1.05 \mathrm{pu}$, which validates the effectiveness of the coordinated charging strategy in voltage profile improvement.

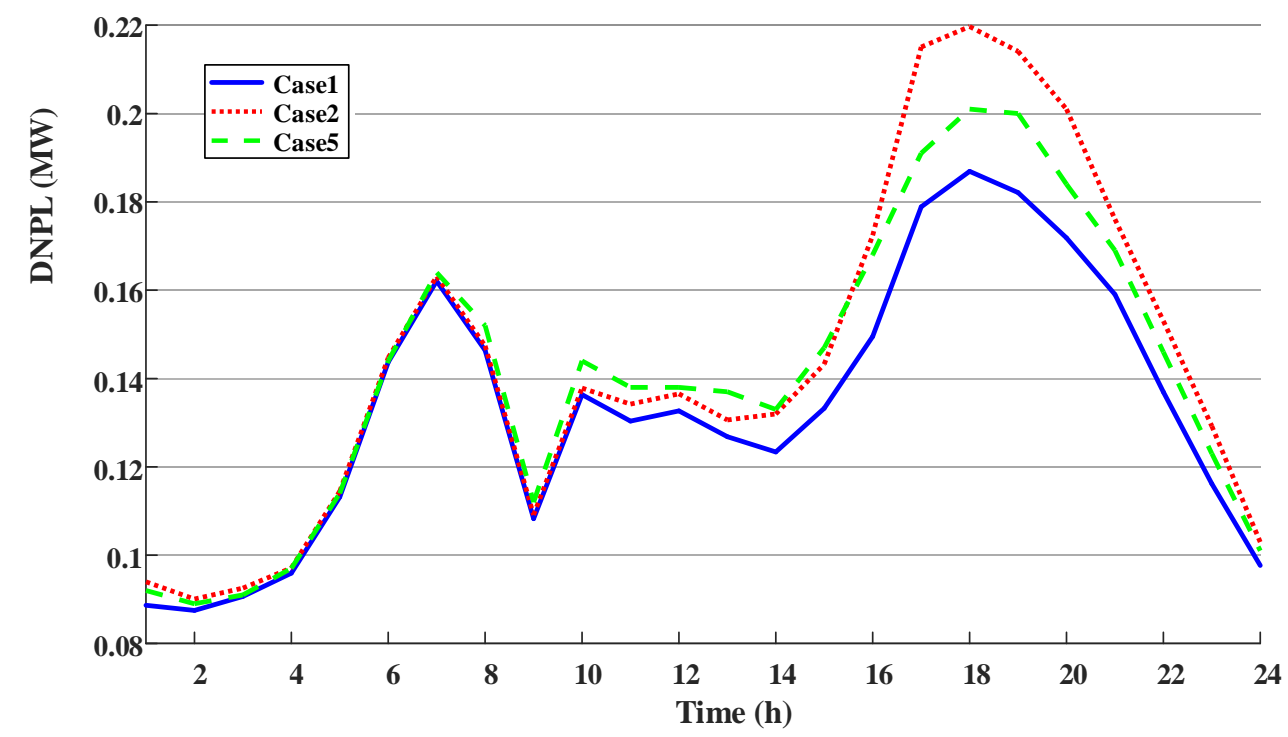

Figure 7. Daily power loss of the distribution network.

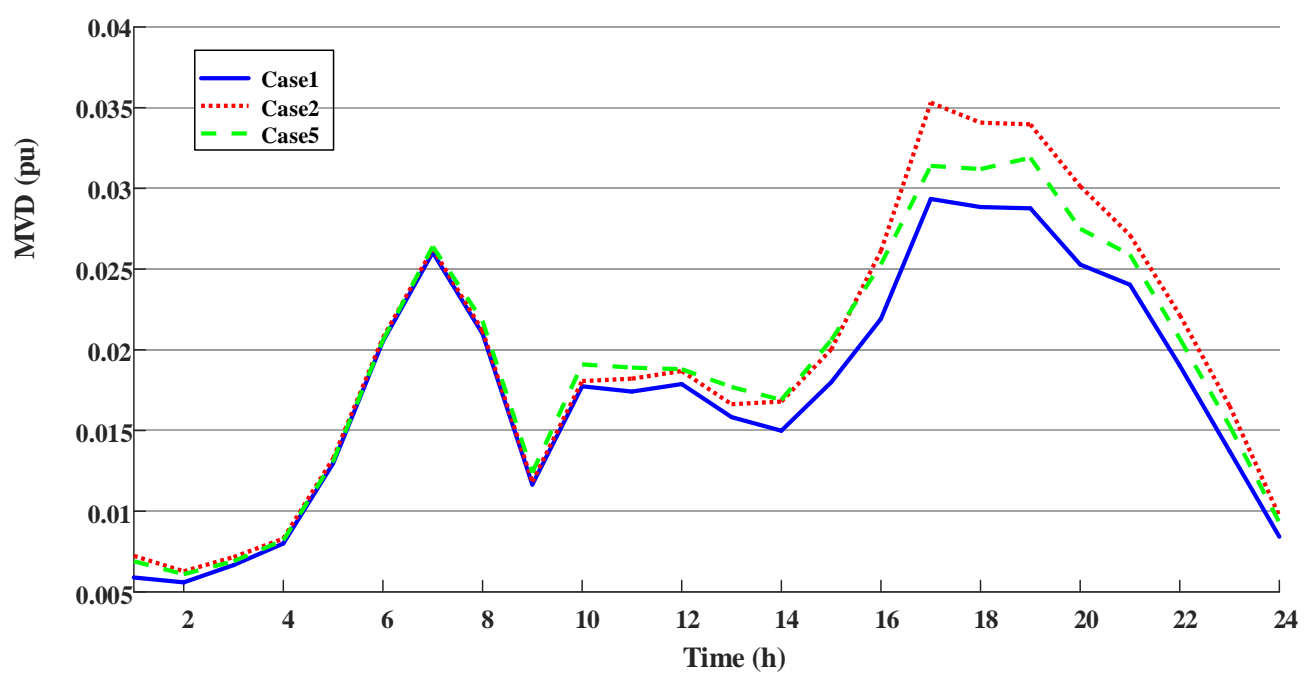

Figure 8. Maximal voltage deviation of the distribution network. 


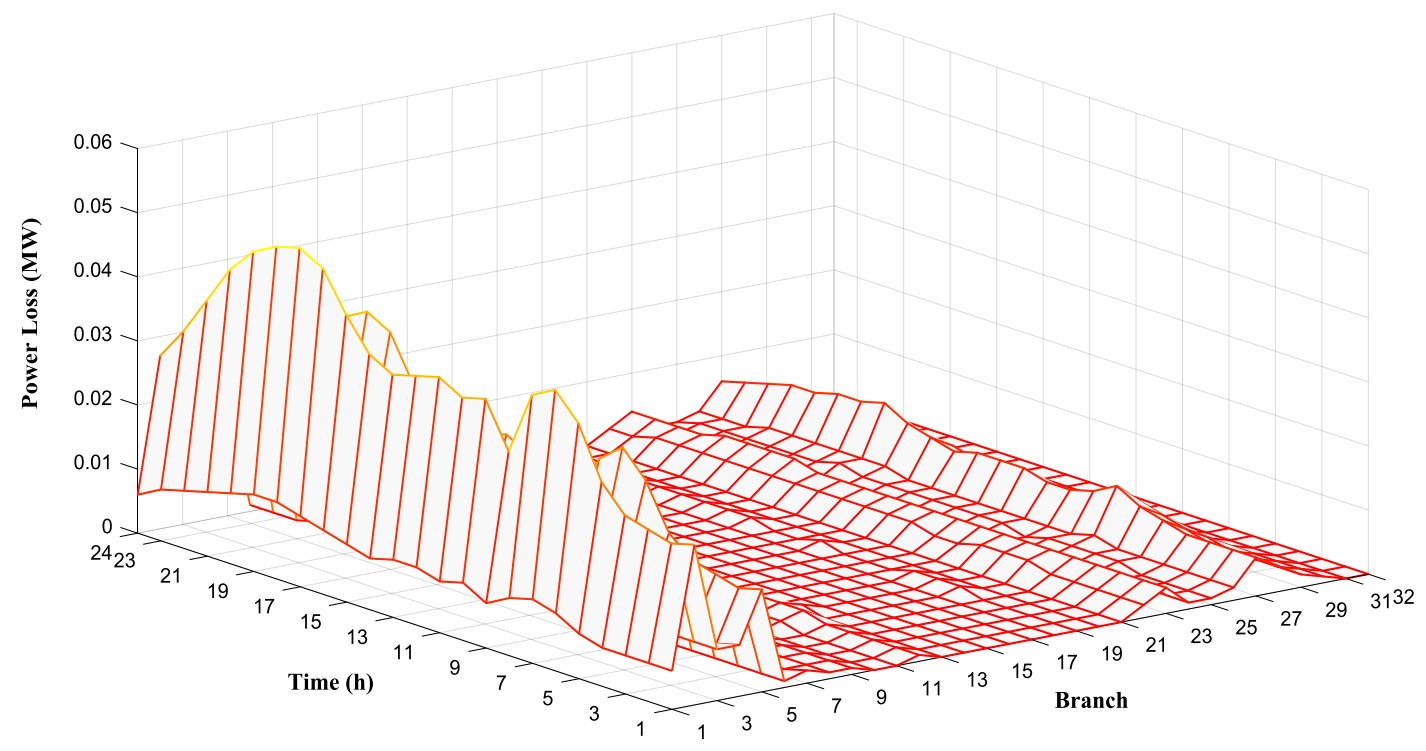

Figure 9. Power loss of the distribution network.

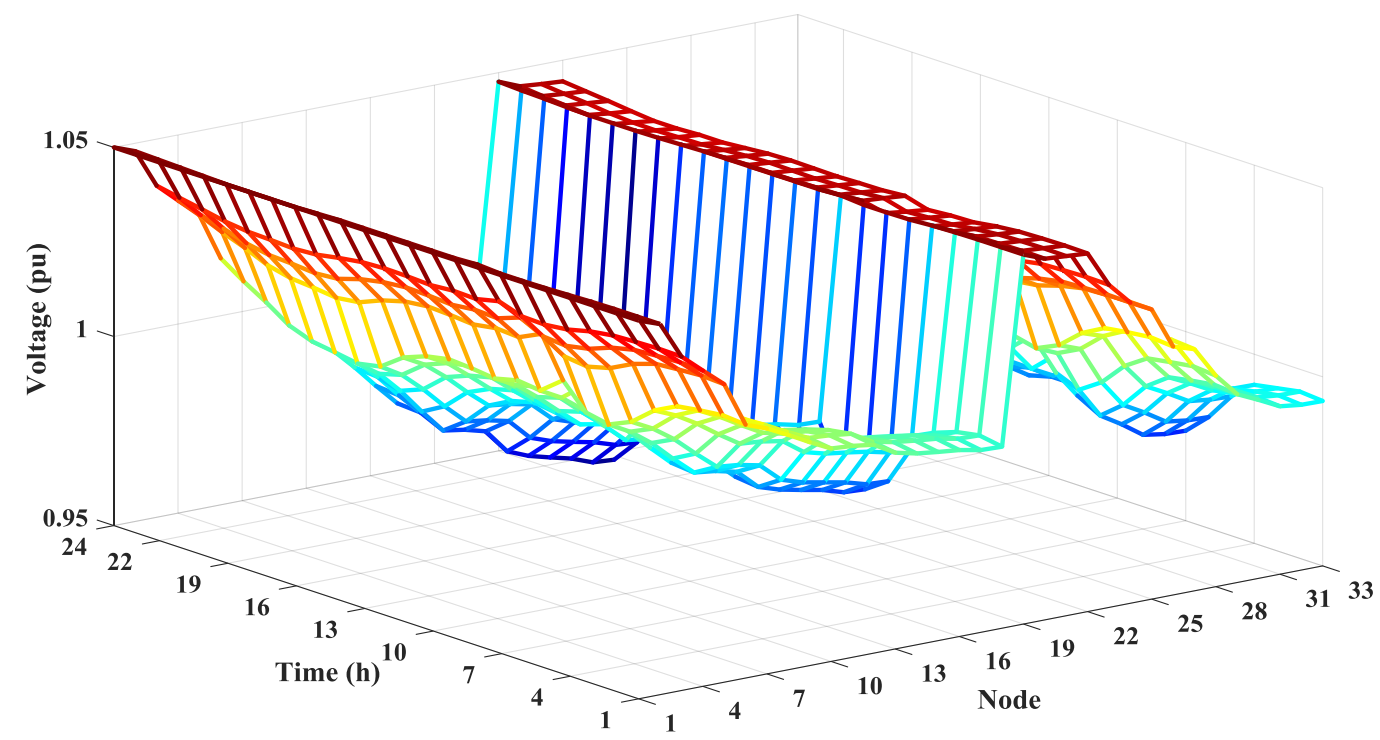

Figure 10. Node voltage of the distribution network.

\subsubsection{Charging Load Analysis}

The optimal charging load of PEVs in different locations has large differences, as shown in Figure 11. The charging load in RCS is the highest and the peak load occurs from 18:00 to 21:00. This is because most residents are used to charging at home after the last trip. The charging behavior in WCS mostly occurs in 8:00-14:00 and the charging load is smaller than only RCS. The charging load in PCS and OCS is relatively small, which is because once PEVs are fully charged in RCS or WCS, it is enough for the users to finish daily trips. Through the optimal charging, the number of PEVs are properly assigned to different charging stations, which avoids the large-scale concentrated charging at rush hour. 


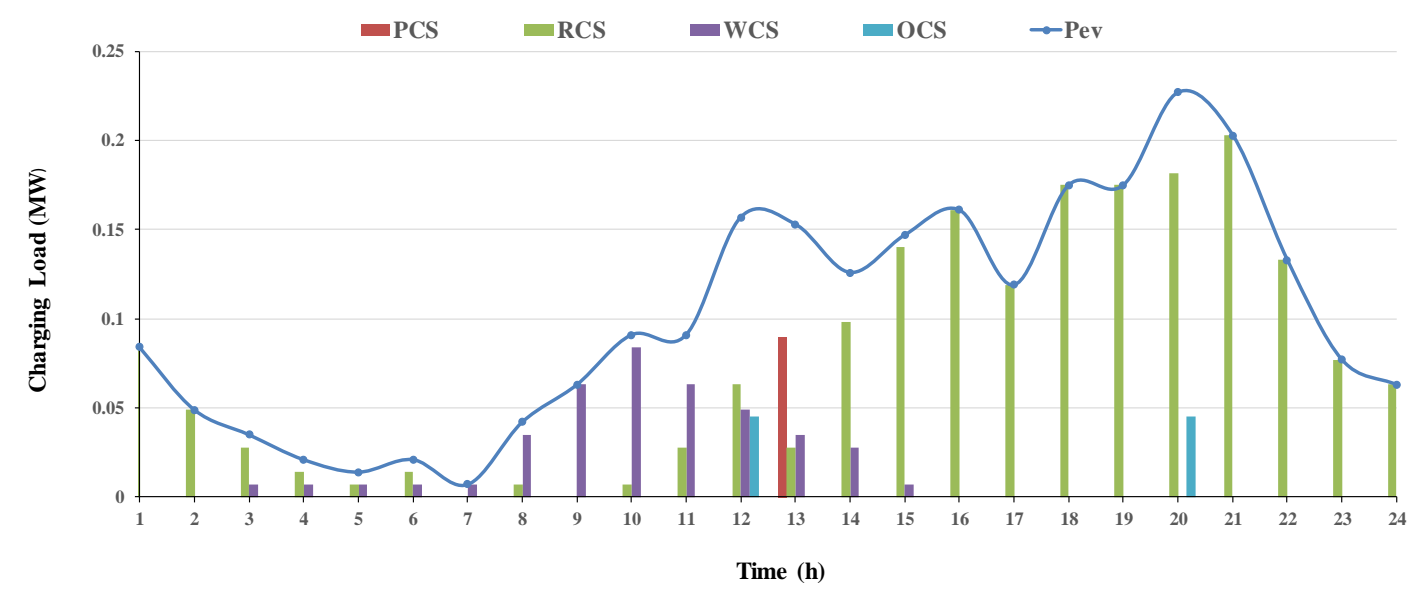

Figure 11. The optimal charging load of PEVs.

\subsubsection{Peak Valley Load Analysis}

The daily load curve of the distribution network in different cases is shown in Figure 12 and the peak valley load values are shown in Table 9. In case 1, the peak-valley difference is 1.101 MW. With the large number of PEVs randomly connected to the distribution network, the fluctuation of the daily load curve is deeply aggravated, and the peak-valley difference in case 2 increases to $1.402 \mathrm{MW}$. Compared with case 2, the load peak valley difference in case 5 is reduced by $12.5 \%$ with the coordinated charging of PEVs. From Figure 12, it can be observed that after the optimal charging, the increased charging load at rush hour can be reduced and transferred to other moments, which shows the effectiveness of the coordinated charging strategy in reducing the peak valley difference.

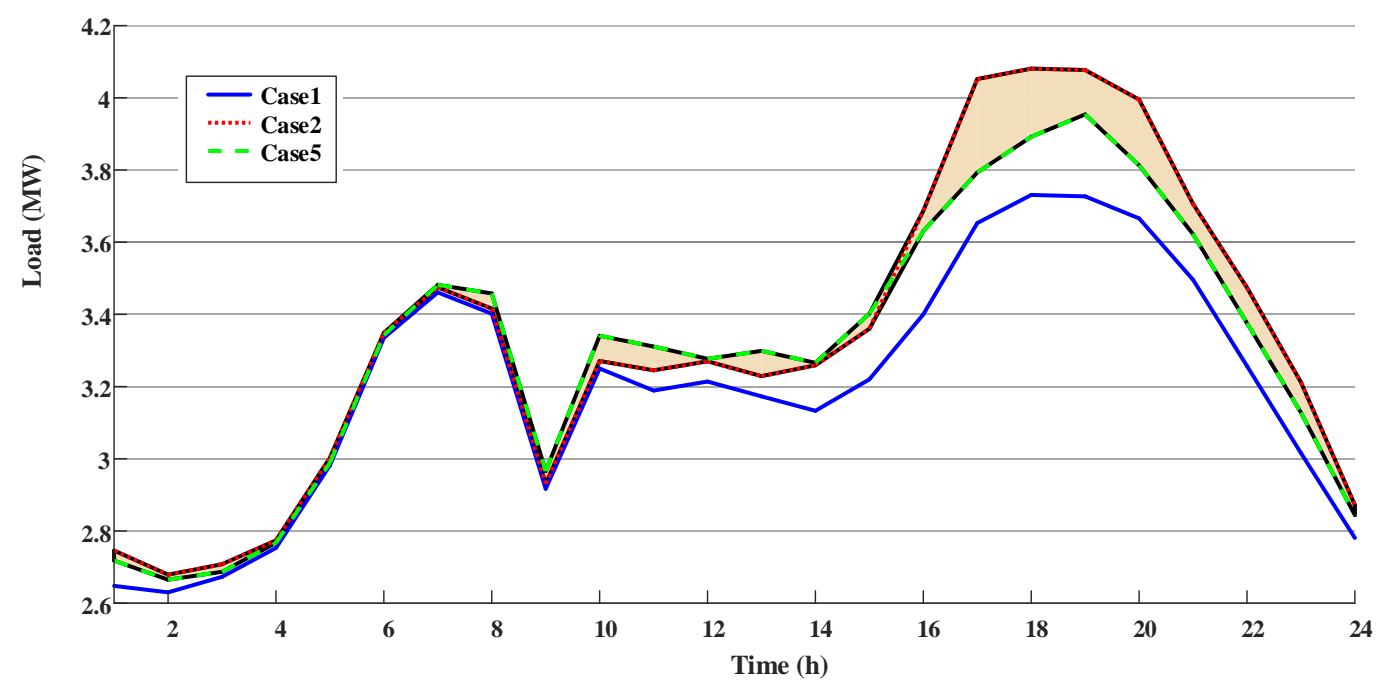

Figure 12. Daily load curve of the distribution network.

Table 9. Peak and valley load values in different cases.

\begin{tabular}{cccc}
\hline Parameters & Case 1 & Case 2 & Case 5 \\
\hline Peak load (MW) & 3.731 & 4.081 & 3.906 \\
Valley load (MW) & 2.63 & 2.679 & 2.679 \\
Peak-valley difference (MW) & 1.101 & 1.402 & 1.227 \\
\hline
\end{tabular}




\section{Conclusions}

This paper proposes a spatial and temporal optimization strategy for PEV charging to mitigate impacts on the distribution network, in which the moving characteristics and charging habits of PEV owners are taken into account. Based on trip chain, the spatial and temporal distribution of PEVs is analyzed and then a stochastic optimization model for PEV charging is established to minimize the distribution network power loss (DNPL) and maximal voltage deviation (MVD). The particle swarm optimization (PSO) algorithm with an embedded power flow program is adopted to solve the optimization problem by shifting the charging load of PEVs in different locations. According to the simulation results, the proposed strategy is feasible and effective. It can reduce power loss and improve voltage profile greatly as well as reduce the peak valley difference. Our future work will consider the demand response measures to meet the charging demand of PEVs.

Author Contributions: L.G. conceived the idea of the topic, formulated the stochastic optimization model, and conducted all programming and the results analysis. W.C. conducted the original data analysis. K.L. was in charge of the main design of the paper and organized the paper with X.L. J.Z. provided insights and additional ideas on presentation. All authors revised and approved the manuscript.

Acknowledgments: This work was supported in part by grants from Key Research and Development Program of Jiangsu Province [grant number BE2015157].

Conflicts of Interest: The authors declare no conflict of interest.

\section{References}

1. Chandrasekhar, A.; Alluri, N.R.; Sudhakaran, M.S.P.; Mok, Y.S.; Kim, S.J. A smart mobile pouch as a biomechanical energy harvester towards self-powered smart wireless power transfer applications. Nanoscale 2017, 9, 9818-9824. [CrossRef] [PubMed]

2. Aravinthan, V.; Jewell, W. Controlled Electric Vehicle Charging for Mitigating Impacts on Distribution Assets. IEEE Trans. Smart Grid 2015, 6, 999-1009. [CrossRef]

3. Luo, Z.W.; Hu, Z.C.; Song, Y.H.; Xu, Z.W.; Lu, H.Y. Optimal coordination of plug-in electric vehicles in power grids with cost-benefit analysis-Part I: Enabling techniques. IEEE Trans. Power Syst. 2013, 28, 3546-3555. [CrossRef]

4. Clement-Nyns, K.; Haesen, E.; Driesen, J. The impact of charging plug-in hybrid electric vehicles on a residential distribution grid. IEEE Trans. Power Syst. 2010, 25, 371-380. [CrossRef]

5. Azhar, U.; Marium, A.; Yousef, M.; Aqib, P.; Essam, A.A. Probabilistic Modeling of Electric Vehicle Charging Pattern Associated with Residential Load for Voltage Unbalance Assessment. Energies 2017, 10, 1351.

6. Jiang, C.; Torquato, R.; Salles, D.; Xu, W. Method to assess the power-quality impact of plug-in electric vehicles. IEEE Trans. Power Deliv. 2014, 29, 958-965. [CrossRef]

7. Leou, R.C.; Su, C.L.; Lu, C.N. Stochastic analyses of electric vehicle charging impacts on distribution network. IEEE Trans. Power Syst. 2014, 29, 1055-1063. [CrossRef]

8. Richardson, P.; Flynn, D.; Keane, A. Optimal charging of electric vehicles in low voltage distribution systems. IEEE Trans. Power Syst. 2012, 27, 268-279. [CrossRef]

9. Gan, L.; Topcu, U.; Low, S.H. Optimal decentralized protocol for electric vehicle charging. IEEE Trans. Power Syst. 2013, 28, 940-951. [CrossRef]

10. Xi, X.; Sioshansi, R. Using Price-Based Signals to Control Plug-in Electric Vehicle Fleet Charging. IEEE Trans. Smart Grid 2014, 5, 1451-1464. [CrossRef]

11. Zakariazadeh, A.; Jadid, S.; Siano, P. Multi-objective scheduling of electric vehicles in smart distribution system. Energy Convers. Manag. 2014, 79, 43-53. [CrossRef]

12. Hoog, J.D.; Alpcan, T.; Brazil, M.; Thomas, D.A.; Mareels, I. Optimal charging of electric vehicles taking distribution network constraints into account. IEEE Trans. Power Syst. 2015, 30, 365-375. [CrossRef]

13. Hu, Z.C.; Qiao, Z.K.; Zhang, H.C.; Song, Y.H. Pricing mechanisms design for guiding electric vehicle charging to fill load valley. Appl. Energy 2016, 178, 155-163. [CrossRef]

14. Jian, L.N.; Zheng, Y.C.; Shao, Z.Y. High efficient valley-filling strategy for centralized coordinated charging of large-scale electric vehicles. Appl. Energy 2017, 186, 46-55. [CrossRef] 
15. Crow, M.; Maigha, M. Cost-Constrained Dynamic Optimal Electric Vehicle Charging. IEEE Trans. Sustain. Energy 2017, 8, 716-724.

16. Chen, L.; Nie, Y.; Zhong, Q. A Model for Electric Vehicle Charging Load Forecasting Based on Trip Chains. Trans. China Electrotech. Soc. 2015, 30, 216-225.

17. U.S. Department of Transportation, Federal Highway Administration. National Household Travel Survey. 2009. Available online: https:/ /nhts.ornl.gov (accessed on 25 April 2018).

18. Gao, F.; Guo, Y.Y.; Chen, J.C. Study on the Travel Attributes of Private Cars on the Basis of Home-Based Trip Chains. J. Transp. Syst. Eng. Inf. Technol. 2011, 11, 132-139.

19. Ullah, I.; Ahmad, R.; Kim, D. A Prediction Mechanism of Energy Consumption in Residential Buildings Using Hidden Markov Model. Energies 2018, 11, 358. [CrossRef]

20. Zhang, B.L.; Sun, Y.T.; Li, B.Q.; Li, J.X. A Modeling Method for the Power Demand of Electric Vehicles Based on Monte Carlo Simulation. In Proceedings of the Asia-Pacific Power \& Energy Engineering Conference, Shanghai, China, 27-29 March 2012; pp. 1-5.

21. Tian, L.T.; Shi, S.L.; Jia, Z. A statistical model for charging power demand of electric vehicles. Power Syst. Technol. 2010, 34, 126-130.

22. Masoum, A.S.; Deilami, S.; Moses, P.S.; Masoum, M.A.S.; Abu-Siada, A. Smart load management of plug-in electric vehicles in distribution and residential networks with charging stations for peak shaving and loss minimization considering voltage regulation. IET Gener. Transm. Distrib. 2011, 5, 877-888. [CrossRef]

23. Knezović, K.; Marinelli, M. Phase-wise enhanced voltage support from electric vehicles in a Danish low-voltage distribution grid. Electr. Power Syst. Res. 2016, 140, 274-283. [CrossRef]

24. Ghosh, D.; Chakraborty, D. A new Pareto set generating method for multi-criteria optimization problems. Oper. Res. Lett. 2014, 42, 514-521. [CrossRef]

25. Cococcioni, M.; Pappalardo, M.; Sergeyev, Y.D. Lexicographic multi-objective linear programming using grossone methodology: Theory and algorithm. Appl. Math. Comput. 2018, 318, 298-311. [CrossRef]

26. Yu, X.; Tan, Y.; Liu, L.; Huang, W. The Optimal Portfolio Model Based on Mean-CvaR with Linear Weighted Sum Method. J. Math. Financ. 2011, 1, 132-134. [CrossRef]

27. Wu, H.B.; Zhuang, H.D.; Zhang, W.; Ding, M. Optimal allocation of microgrid considering economic dispatch based on hybrid weighted bilevel planning method and algorithm improvement. Int. J. Electr. Power Energy Syst. 2016, 75, 28-37. [CrossRef]

28. Zhao, X.; Zhao, C.Y.; Jia, X.F.; Li, G.Y. Fuzzy synthetic evaluation of power quality based on changeable weight. Power Syst. Technol. 2005, 29, 11-16.

29. Liu, Y.X.; Liu, S.; Wang, W.Y. Computation of Weight in AHP and Its Application. J. Shenyang Univ. 2014, 26, 372-375.

30. Dorigo, M.; Blum, C. Ant colony optimization theory: A survey. Theor. Comput. Sci. 2005, 344, $243-278$. [CrossRef]

31. Holland, J.H. Building Blocks, Cohort Genetic Algorithms, and Hyperplane-Defined Functions. Evol. Comput. 2000, 8, 373-391. [CrossRef] [PubMed]

32. Kenndy, J.; Eberhart, R.C. Particle swarm optimization. In Proceedings of the IEEE International Conference on Neutral Networks, Perth, Australia, 27 November-1 December 1995; Volume 4, pp. 1942-1948.

33. Liu, P. Research on Load Modeling of Electric Vehicles and Its Applications; School of Electrical Engineering and Automation, Harbin Institute of Technology: Harbin, China, 2012.

34. Luo, Z.W.; Hu, Z.C.; Song, Y.H.; Xu, Z.W.; Lu, H.Y. Optimal coordination of plug-in electric vehicles in power grids with cost-benefit analysis-Part II: A Case Study in China. IEEE Trans. Power Syst. 2013, 28, 3556-3565. [CrossRef]

35. Li, J.; Yang, L.; Liu, J.L.; Yang, D.L.; Zhang, C. Multi-objective reactive power optimization based on adaptive chaos particle swarm optimization algorithm. Power Syst. Prot. Control 2011, 39, 26-31.

36. Li, L.H. Research on Power System Reactive Power Optimization Based on Improved Particle Swarm Optimization; Taiyuan University of Technology: Taiyuan, China, 2012.

(C) 2018 by the authors. Licensee MDPI, Basel, Switzerland. This article is an open access article distributed under the terms and conditions of the Creative Commons Attribution (CC BY) license (http://creativecommons.org/licenses/by/4.0/). 